\title{
Pacific
}

Journal of

Mathematics

ENERGY IDENTITY FOR THE MAPS FROM A SURFACE WITH TENSION FIELD BOUNDED IN $L^{p}$

\section{JIAYU AND ZHU XIANGRONG}




\title{
ENERGY IDENTITY FOR THE MAPS FROM A SURFACE WITH TENSION FIELD BOUNDED IN $L^{p}$
}

\author{
LI JIAYU AND ZHU XIANGRONG
}

Let $M$ be a closed Riemannian surface and $u_{n}$ a sequence of maps from $M$ to Riemannian manifold $N$ satisfying

$$
\sup _{n}\left(\left\|\nabla u_{n}\right\|_{L^{2}(M)}+\left\|\tau\left(u_{n}\right)\right\|_{L p(M)}\right) \leq \Lambda
$$

for some $p>1$, where $\tau\left(u_{n}\right)$ is the tension field of the mapping $u_{n}$.

For a general target manifold $N$, if $p \geq \frac{6}{5}$, we prove the energy identity and the neckless property during blowing up.

\section{Introduction}

Let $(M, g)$ be a closed Riemannian manifold and $(N, h)$ be a Riemannian manifold without boundary. For a mapping $u$ from $M$ to $N$ in $W^{1,2}(M, N)$, the energy density of $u$ is defined by

$$
e(u)=\frac{1}{2}|d u|^{2}=\operatorname{Trace}_{g} u^{*} h,
$$

where $u^{*} h$ is the pull-back of the metric tensor $h$.

The energy of the mapping $u$ is defined as

$$
E(u)=\int_{M} e(u) d V,
$$

where $d V$ is the volume element of $(M, g)$.

A map $u \in C^{1}(M, N)$ is called harmonic if it is a critical point of the energy $E$.

By the Nash embedding theorem we know that $(N, h)$ can be isometrically into a Euclidean space $\mathbb{R}^{K}$ with some positive integer $K$. Then $(N, h)$ may be considered as a submanifold of $\mathbb{R}^{K}$ with the metric induced from the Euclidean metric. Thus a map $u \in C^{1}(M, N)$ can be considered as a map of $C^{1}\left(M, \mathbb{R}^{K}\right)$ whose image lies in $N$. In this sense we can get the Euler-Lagrange equation

$$
\triangle u=A(u)(d u, d u) .
$$

The research was supported by NSFC grants number 11071236, 11131007 and 11101372 and by PCSIRT.

MSC2010: 58E20.

Keywords: energy identity, tension field, neckless. 
The tension field $\tau(u)$ is defined by

$$
\tau(u)=\triangle_{M} u-A(u)(d u, d u),
$$

where $A(u)(d u, d u)$ is the second fundamental form of $N$ in $\mathbb{R}^{K}$. So $u$ being harmonic means that $\tau(u)=0$.

The harmonic mappings are of special interest when $M$ is a Riemann surface. Consider a sequence of mappings $u_{n}$ from Riemann surface $M$ to $N$ with bounded energies. It is clear that $u_{n}$ converges weakly to $u$ in $W^{1,2}(M, N)$ for some $u$ in $W^{1,2}(M, N)$. But in general, it may not converge strongly in $W^{1,2}(M, N)$. When $\tau\left(u_{n}\right)=0$, that is, when $u_{n}$ are all harmonic, Parker [1996] proved that the lost energy is exactly the sum of some harmonic spheres, which are defined as harmonic mappings from $S^{2}$ to $N$. This result is called the energy identity. Also he proved that the images of these harmonic spheres and $u(M)$ are connected, that is, there is no neck during blowing up.

When $\tau\left(u_{n}\right)$ is bounded in $L^{2}$, the energy identity was proved in [Qing 1995] for the sphere, and in [Ding and Tian 1995] and [Wang 1996] for a general target manifold. Qing and Tian [1997] proved there is no neck during blowing up. For the heat flow of harmonic mappings, the results can also be found in [Topping 2004a; $2004 \mathrm{~b}$ ]. When the target manifold is a sphere, we proved the energy identity in [Li and Zhu 2011] for a sequence of mappings with tension fields bounded in $L \ln ^{+} L$, using good observations from [Lin and Wang 2002]. On the other hand, in the same paper we constructed a sequence of mappings with tension fields bounded in $L \ln ^{+} L$ such that there is a positive neck during blowing up. In [Zhu 2012] the neckless property during blowing up was proved for a sequence of maps $u_{n}$ with

$$
\lim _{\delta \rightarrow 0} \sup _{n} \sup _{B(x, \delta) \subset D_{1}}\left\|\tau\left(u_{n}\right)\right\|_{L \ln ^{+}} L(B(x, \delta))=0 .
$$

In this paper we prove the energy identity and neckless property during blowing up of a sequence of maps $u_{n}$ with $\tau\left(u_{n}\right)$ bounded in $L^{p}$ for some $p \geq \frac{6}{5}$, for a general target manifold.

When $\tau\left(u_{n}\right)$ is bounded in $L^{p}$ for some $p>1$, the small energy regularity proved in [Ding and Tian 1995] implies that $u_{n}$ converges strongly in $W^{1,2}(M, N)$ outside a finite set of points. For simplicity of exposition, it is no matter to assume that $M$ is the unit disk $D_{1}=D(0,1)$ and there is only one singular point at 0 .

In this paper we prove the following theorem.

Theorem 1. Let $\left\{u_{n}\right\}$ be a sequence of mappings from $D_{1}$ to $N$ in $W^{1,2}\left(D_{1}, N\right)$ with tension field $\tau\left(u_{n}\right)$. If

(a) $\left\|u_{n}\right\|_{W^{1,2}\left(D_{1}\right)}+\left\|\tau\left(u_{n}\right)\right\|_{L^{p}\left(D_{1}\right)} \leq \Lambda$ for some $p \geq \frac{6}{5}$,

(b) $u_{n} \rightarrow u$ strongly in $W^{1,2}\left(D_{1} \backslash\{0\}, \mathbb{R}^{K}\right)$ as $n \rightarrow \infty$, 
then there exists a subsequence of $\left\{u_{n}\right\}$ (we still denote it by $\left\{u_{n}\right\}$ ) and some nonnegative integer $k$ so that for any $i=1, \ldots, k$, there exist points $x_{n}^{i}$, positive numbers $r_{n}^{i}$ and a nonconstant harmonic sphere $w^{i}$ (which we view as a map from $\left.\mathbb{R}^{2} \cup\{\infty\} \rightarrow N\right)$ such that:

(1) $x_{n}^{i} \rightarrow 0, r_{n}^{i} \rightarrow 0$ as $n \rightarrow \infty$.

(2) $\lim _{n \rightarrow \infty}\left(\frac{r_{n}^{i}}{r_{n}^{j}}+\frac{r_{n}^{j}}{r_{n}^{i}}+\frac{\left|x_{n}^{i}-x_{n}^{j}\right|}{r_{n}^{i}+r_{n}^{j}}\right)=\infty$ for any $i \neq j$.

(3) $w^{i}$ is the weak limit or strong limit of $u_{n}\left(x_{n}^{i}+r_{n}^{i} x\right)$ in $W_{L o c}^{1,2}\left(\mathbb{R}^{2}, N\right)$.

(4) Energy identity: We have

$$
\lim _{n \rightarrow \infty} E\left(u_{n}, D_{1}\right)=E\left(u, D_{1}\right)+\sum_{i=1}^{k} E\left(w^{i}\right) .
$$

(5) Neckless property: The image $u\left(D_{1}\right) \cup \bigcup_{i=1}^{k} w^{i}\left(\mathbb{R}^{2}\right)$ is a connected set.

This paper is organized as follows. In Section 2 we state some basic lemmas and some standard arguments in the blow-up analysis.

In Section 3 and Section 4 we prove Theorem 1. In the proof, we use delicate analysis on the difference between normal energy and tangential energy. The energy identity is proved in Section 3 and the neckless property is proved in Section 4.

Throughout this paper, the letter $C$ denotes a positive constant that depends only on $p, \Lambda$ and the target manifold $N$ and may vary in different places. We also don't distinguish between a sequence and one of its subsequences.

\section{Some basic lemmas and standard arguments}

We recall the regular theory for a mapping with small energy on the unit disk and tension field in $L^{p} \quad(p>1)$.

Lemma 2. Let $\bar{u}$ be the mean value of $u$ on the disk $D_{1 / 2}$. There exists a positive constant $\epsilon_{N}$ that depends only on the target manifold such that if $E\left(u, D_{1}\right) \leq \epsilon_{N}^{2}$ then

$$
\|u-\bar{u}\|_{W^{2, p}\left(D_{1 / 2}\right)} \leq C\left(\|\nabla u\|_{L^{2}\left(D_{1}\right)}+\|\tau(u)\|_{p}\right)
$$

where $p>1$.

As a consequence of (2-1) and the Sobolev embedding $W^{2, p}\left(\mathbb{R}^{2}\right) \subset C^{0}\left(\mathbb{R}^{2}\right)$, we have

$$
\|u\|_{O s c\left(D_{1 / 2}\right)}=\sup _{x, y \in D_{1 / 2}}|u(x)-u(y)| \leq C\left(\|\nabla u\|_{L^{2}\left(D_{1}\right)}+\|\tau(u)\|_{p}\right) .
$$


Remarks. - In [Ding and Tian 1995] this lemma is proved for the mean value of $u$ on the unit disk. Note that

$$
\left|\frac{\int_{D_{1}} u(x) d x}{\left|D_{1}\right|}-\frac{\int_{D_{1 / 2}} u(x) d x}{\left|D_{1 / 2}\right|}\right| \leq C\|\nabla u\|_{L^{2}\left(D_{1}\right)} .
$$

So we can use the mean value of $u$ on $D_{1 / 2}$ in this lemma.

- Suppose we have a sequence of mappings $u_{n}$ from the unit disk $D_{1}$ to $N$ with $\left\|u_{n}\right\|_{W^{1,2}\left(D_{1}\right)}+\left\|\tau\left(u_{n}\right)\right\|_{L^{p}\left(D_{1}\right)} \leq \Lambda$ for some $p>1$.

A point $x \in D_{1}$ is called an energy concentration point (blow-up point) if for any $r$ such that $D(x, r) \subset D_{1}$, we have

$$
\sup _{n} E\left(u_{n}, D(x, r)\right)>\epsilon_{N}^{2},
$$

where $\epsilon_{N}$ is given in this lemma. If $x \in D_{1}$ isn't an energy concentration point, we can find a positive number $\delta$ such that

$$
E\left(u_{n}, D(x, \delta)\right) \leq \epsilon_{N}^{2} \text { for all } n .
$$

Then it follows from Lemma 2 that we have a uniformly $W^{2, p}(D(x, \delta / 2))$ bound for $u_{n}$. Because $W^{2, p}$ is compactly embedded in $W^{1,2}$, there is a subsequence of $u_{n}$ (still denoted by $u_{n}$ ) and $u \in W^{2, p}(D(x, \delta / 2)$ ) such that

$$
\lim _{n \rightarrow \infty} u_{n}=u \quad \text { in } W^{1,2}(D(x, \delta / 2)) \text {. }
$$

So $u_{n}$ converges to $u$ strongly in $W^{1,2}\left(D_{1}\right)$ outside a finite set of points.

Under the assumptions of our theorem, by the standard blow-up argument, that is by repeatedly rescaling $u_{n}$ in a suitable way, we can obtain some nonnegative integer $k$ so that for any $i=1, \ldots, k$, there exist a point $x_{n}^{i}$, a positive number $r_{n}^{i}$ and a nonconstant harmonic sphere $w^{i}$ satisfying (1), (2) and (3) of Theorem 1. By the standard induction argument in [Ding and Tian 1995] we only need to prove the theorem in the case where there is only one bubble.

In that case we can assume that $w$ is the strong limit of the sequence $u_{n}\left(x_{n}+r_{n} x\right)$ in $W_{\text {Loc }}^{1,2}\left(\mathbb{R}^{2}\right)$. We may assume that $x_{n}=0$. Set $w_{n}(x)=u_{n}\left(r_{n} x\right)$.

As

$$
\lim _{\delta \rightarrow 0} \lim _{n \rightarrow \infty} E\left(u_{n}, D_{1} \backslash D_{\delta}\right)=E\left(u, D_{1}\right),
$$

the energy identity is equivalent to

$$
\lim _{\delta \rightarrow 0} \lim _{n \rightarrow \infty} \lim _{R \rightarrow \infty} E\left(u_{n}, D_{\delta} \backslash D_{r_{n} R}\right)=0 .
$$

To prove the sets $u\left(D_{1}\right)$ and $w\left(\mathbb{R}^{2} \cup \infty\right)$ are connected, it is enough to show that

$$
\lim _{\delta \rightarrow 0} \lim _{n \rightarrow \infty} \lim _{R \rightarrow \infty} \sup _{x, y \in D_{\delta} \backslash D_{r_{n} R}}\left|u_{n}(x)-u_{n}(y)\right|=0 .
$$




\section{Energy identity}

In this section, we prove the energy identity for a general target manifold when $p \geq \frac{6}{5}$.

Assume that there is only one bubble $w$ which is the strong limit of $u_{n}\left(r_{n} \cdot\right)$ in $W_{L o c}^{1,2}\left(\mathbb{R}^{2}\right)$. Let $\epsilon_{N}$ be the constant in Lemma 2. By the standard argument of blow-up analysis we can assume that, for any $n$,

$$
E\left(u_{n}, D_{r_{n}}\right)=\sup _{\substack{r \leq r_{n} \\ D(x, r) \subseteq D_{1}}} E\left(u_{n}, D(x, r)\right)=\frac{1}{4} \epsilon_{N}^{2} .
$$

Lemma 3 [Ding and Tian 1995]. If $\tau\left(u_{n}\right)$ is bounded in $L^{p}$ for some $p>1$, then the tangential energy on the neck domain is zero, that is,

$$
\lim _{\delta \rightarrow 0} \lim _{R \rightarrow \infty} \lim _{n \rightarrow \infty} \int_{D_{\delta} \backslash D_{r_{n} R}}|x|^{-2}\left|\partial_{\theta} u\right|^{2} d x=0 .
$$

Proof. The proof is the same as in [Ding and Tian 1995], so we only sketch it.

For any $\epsilon>0$, take $\delta, R$ such that, for any $n$,

$$
E\left(u, D_{4 \delta}\right)+E\left(w, \mathbb{R}^{2} \backslash D_{R}\right)+\delta^{4(p-1) / p}<\epsilon^{2} .
$$

We may suppose that $r_{n} R=2^{-j_{n}}, \delta=2^{-j_{0}}$. When $n$ is big enough we have, for any $j_{0} \leq j \leq j_{n}$,

$$
E\left(u_{n}, D_{2^{1-j}} \backslash D_{2^{-j}}\right)<\epsilon^{2} .
$$

For any $j$, set

$$
h_{n}\left(2^{-j}\right)=\frac{1}{2 \pi} \int_{S^{1}} u_{n}\left(2^{-j}, \theta\right) d \theta
$$

and

$$
h_{n}(t)=h_{n}\left(2^{-j}\right)+\left(h_{n}\left(2^{1-j}\right)-h_{n}\left(2^{-j}\right)\right) \frac{\ln \left(2^{j} t\right)}{\ln 2}, \quad t \in\left[2^{-j}, 2^{1-j}\right] .
$$

It is easy to check that

$$
\frac{d^{2} h_{n}(t)}{d t^{2}}+\frac{1}{t} \frac{d h_{n}(t)}{d t}=0, \quad t \in\left[2^{-j}, 2^{1-j}\right] .
$$

Consider $h_{n}(x)=h_{n}(|x|)$ as a map from $\mathbb{R}^{2}$ to $\mathbb{R}^{K}$, then $\triangle h_{n}=0$ in $\mathbb{R}^{2}$. Setting $P_{j}=D_{2^{1-j}} \backslash D_{2^{-j}}$ we have

$$
\triangle\left(u_{n}-h_{n}\right)=\triangle u_{n}-\triangle h_{n}=\triangle u_{n}=A\left(u_{n}\right)+\tau\left(u_{n}\right), \quad x \in P_{j} .
$$

Taking the inner product of this equation with $u_{n}-h_{n}$ and integrating over $P_{j}$, we get that

$$
\int_{P_{j}}\left|\nabla\left(u_{n}-h_{n}\right)\right|^{2} d x=-\int_{P_{j}}\left(u_{n}-h_{n}\right)\left(A\left(u_{n}\right)+\tau\left(u_{n}\right)\right) d x+\int_{\partial P_{j}}\left(u_{n}-h_{n}\right)\left(u_{n}-h_{n}\right)_{r} d s .
$$


Note that by definition, $h_{n}\left(2^{-j}\right)$ is the mean value of $\left\{2^{-j}\right\} \times S^{1}$ and $\left(h_{n}\right)_{r}$ is independent of $\theta$. So the integral of $\left(u_{n}-h_{n}\right)\left(h_{n}\right)_{r}$ on $\partial P_{j}$ vanishes.

When $j_{0}<j<j_{n}$, by Lemma 2 we have

$$
\begin{aligned}
\left\|u_{n}-h_{n}\right\|_{C^{0}\left(P_{j}\right)} & \leq\left\|u_{n}-h_{n}\left(2^{-j}\right)\right\|_{C^{0}\left(P_{j}\right)}+\left\|u_{n}-h_{n}\left(2^{1-j}\right)\right\|_{C^{0}\left(P_{j}\right)} \\
& \leq 2\left\|u_{n}\right\|_{O s c\left(P_{j}\right)} \\
& \leq C\left(\left\|\nabla u_{n}\right\|_{L^{2}\left(P_{j-1} \cup P_{j} \cup P_{j+1}\right)}+2^{2(1-p) j / p}\left\|\tau\left(u_{n}\right)\right\|_{p}\right) \\
& \leq C\left(\epsilon+2^{-2(p-1) j / p}\right) \\
& \leq C\left(\epsilon+\delta^{2(p-1) / p}\right) \leq C \epsilon .
\end{aligned}
$$

Summing over $j$ for $j_{0}<j<j_{n}$ gives

$$
\begin{aligned}
\int_{D_{\delta} \backslash D_{2 r_{n} R}} & \left|\nabla\left(u_{n}-h_{n}\right)\right|^{2} d x \\
= & \sum_{j_{0}<j<j_{n}} \int_{P_{j}}\left|\nabla\left(u_{n}-h_{n}\right)\right|^{2} d x \\
\leq & \sum_{j_{0}<j<j_{n}} \int_{P_{j}}\left|u_{n}-h_{n}\right|\left(\left|A\left(u_{n}\right)\right|+\left|\tau\left(u_{n}\right)\right|\right) d x \\
& \quad+\sum_{j_{0}<j<j_{n}} \int_{\partial P_{j}}\left(u_{n}-h_{n}\right)\left(u_{n}-h_{n}\right)_{r} d s \\
\leq & C \epsilon\left(\int_{D_{2 \delta} \backslash D_{2 r_{n} R}}\left(\left|\nabla u_{n}\right|^{2}+\left|\tau\left(u_{n}\right)\right|\right) d x+\int_{\partial D_{2 \delta} \cup \partial D_{2 r_{n} R}}\left|\nabla u_{n}\right| d s\right) \\
\leq & C \epsilon\left(\int_{D_{2 \delta} \backslash D_{2 r_{n} R}}\left|\nabla u_{n}\right|^{2} d x+\delta^{2(p-1) / p}+\epsilon\right) \leq C \epsilon .
\end{aligned}
$$

Here we use the inequality

$$
\int_{\partial D_{2 \delta} \cup \partial D_{2 r_{n} R}}\left|\nabla u_{n}\right| d s \leq C \epsilon
$$

which can be derived from the Sobolev trace embedding theorem.

As $h_{n}(x)$ is independent of $\theta$, it can be shown that

$$
\int_{D_{2 \delta} \backslash D_{2 r_{n} R}}|x|^{-2}\left|\partial_{\theta} u_{n}\right|^{2} d x \leq \int_{D_{2 \delta} \backslash D_{2 r_{n} R}}\left|\nabla\left(u_{n}-h_{n}\right)\right|^{2} d x \leq C \epsilon,
$$

so this lemma is proved.

It is left to show that the normal energy on the neck domain also equals to zero. We need the following equality.

Lemma 4 (Pohozaev equality [Lin and Wang 1998, Lemma 2.4, page 374]). Let $u$ be a solution to

$$
\triangle u+A(u)(d u, d u)=\tau(u) .
$$


Then

$$
\int_{\partial D_{t}}\left(\left|\partial_{r} u\right|^{2}-r^{-2}\left|\partial_{\theta} u\right|^{2}\right) d s=\frac{2}{t} \int_{D_{t}} \tau \cdot(x \nabla u) d x .
$$

As a direct corollary, by integrating over $[0, \delta]$, we have

$$
\int_{D_{\delta}}\left(\left|\partial_{r} u\right|^{2}-r^{-2}\left|\partial_{\theta} u\right|^{2}\right) d x=\int_{0}^{\delta} \frac{2}{t} \int_{D_{t}} \tau \cdot(x \nabla u) d x d t .
$$

Proof. Multiplying both sides of the equation by $x \nabla u$ and integrating over $D_{t}$, we get

$$
\int_{D_{t}}|\nabla u|^{2} d x-t \int_{\partial D_{t}}\left|\partial_{r} u\right|^{2} d s+\frac{1}{2} \int_{D_{t}} x \nabla|\nabla u|^{2} d x=-\int_{D_{t}} \tau \cdot(x \nabla u) d x .
$$

Note that

$$
\frac{1}{2} \int_{D_{t}} x \nabla|\nabla u|^{2} d x=-\int_{D_{t}}|\nabla u|^{2} d x+\frac{t}{2} \int_{\partial D_{t}}|\nabla u|^{2} d s
$$

Hence,

$$
\int_{\partial D_{t}}\left(\left|\partial_{r} u\right|^{2}-\frac{1}{2}|\nabla u|^{2}\right) d s=\frac{1}{t} \int_{D_{t}} \tau \cdot(x \nabla u) d x .
$$

As $|\nabla u|^{2}=\left|\partial_{r} u\right|^{2}+r^{-2}\left|\partial_{\theta} u\right|^{2}$, we have proved this lemma.

Now we use this equality to estimate the normal energy on the neck domain. We prove the following lemma.

Lemma 5. If $\tau\left(u_{n}\right)$ is bounded in $L^{p}$ for some $p \geq \frac{6}{5}$, then for $\delta$ small enough we have

$$
\left|\int_{D_{\delta}}\left(\left|\partial_{r} u_{n}\right|^{2}-|x|^{-2}\left|\partial_{\theta} u\right|^{2}\right) d x\right| \leq C \delta^{(p-1) / p},
$$

where $C$ depends on $p, \Lambda$, the target manifold $N$ and the bubble $w$.

Proof. Take $\psi \in C_{0}^{\infty}\left(D_{2}\right)$ satisfying $\psi=1$ in $D_{1}$, then

$$
\triangle\left(\psi u_{n}\right)=\psi A\left(u_{n}\right)\left(d u_{n}, d u_{n}\right)+\psi \tau_{n}+2 \nabla \psi \nabla u_{n}+u_{n} \Delta \psi .
$$

Set $g_{n}=\psi A\left(u_{n}\right)\left(d u_{n}, d u_{n}\right)+\psi \tau_{n}+2 \nabla \psi \nabla u_{n}+u_{n} \Delta \psi$. When $|x|<1$,

$$
\partial_{i} u_{n}(x)=R_{i} * g_{n}(x)=\int \frac{x_{i}-y_{i}}{|x-y|^{2}} g_{n}(y) d y .
$$

Let $\Phi_{n}$ be the Newtonian potential of $\psi \tau_{n}$, then $\Delta \Phi_{n}=\psi \tau_{n}$. The corresponding Pohozaev equality is

$$
\int_{D_{\delta}}\left(\left|\partial_{r} \Phi_{n}\right|^{2}-r^{-2}\left|\partial_{\theta} \Phi_{n}\right|^{2}\right) d x=\int_{0}^{\delta} \frac{2}{t} \int_{D_{t}} \psi \tau_{n} \cdot\left(x \nabla \Phi_{n}\right) d x d t .
$$


Here

$$
\partial_{i} \Phi_{n}(x)=R_{i} *\left(\psi \tau_{n}\right)(x)=\int \frac{x_{i}-y_{i}}{|x-y|^{2}}\left(\psi \tau_{n}\right)(y) d y .
$$

As $\tau_{n}$ is bounded in $L^{p} \quad(p>1)$, we have

$\int_{D_{\delta}}\left|\nabla \Phi_{n}\right|^{2} d x \leq C \delta^{4(p-1) / p}\left\|\nabla \Phi_{n}\right\|_{2 p /(2-p)}^{2} \leq C \delta^{4(p-1) / p}\left\|\tau_{n}\right\|_{p}^{2} \leq C \delta^{4(p-1) / p}$.

By (3-7), it can be shown that for any $\delta>0$,

$$
\left|\int_{0}^{\delta} \frac{1}{t} \int_{D_{t}} \psi \tau_{n} \cdot\left(x \nabla \Phi_{n}\right) d x d t\right| \leq \int_{D_{\delta}}\left|\nabla \Phi_{n}\right|^{2} d x \leq C \delta^{4(p-1) / p} .
$$

For $\delta$ small enough, we have

$$
\begin{aligned}
& \left|\int_{D_{\delta}}\left(\left|\partial_{r} u_{n}\right|^{2}-r^{-2}\left|\partial_{\theta} u_{n}\right|^{2}\right) d x\right| \\
& \quad=\left|\int_{0}^{\delta} \frac{2}{t} \int_{D_{t}} \tau_{n} \cdot\left(x \nabla u_{n}\right) d x d t\right| \\
& \quad \leq 2\left|\int_{0}^{\delta} \frac{1}{t} \int_{D_{t}} \tau_{n} \cdot\left(x \nabla \Phi_{n}\right) d x d t\right|+2 \int_{0}^{\delta} \frac{1}{t} \int_{D_{t}}\left|x \tau_{n}\right|\left|\nabla\left(u_{n}-\Phi_{n}\right)(x)\right| d x d t \\
& \leq C \delta^{4(p-1) / p}+2 \int_{D_{\delta}}\left|x \tau_{n}\right|\left|\nabla\left(u_{n}-\Phi_{n}\right)(x)\right|\left(\int_{|x|}^{\delta} \frac{1}{t} d t\right) d x \\
& \quad \leq C \delta^{4(p-1) / p}+2 \int_{D_{\delta}}\left|\tau_{n}\right|\left|\nabla\left(u_{n}-\Phi_{n}\right)(x)\right||x| \ln \frac{1}{|x|} d x .
\end{aligned}
$$

For any $j>0$, set $\varphi_{j}(x)=\psi\left(\frac{x}{2^{2-j} \delta}\right)-\psi\left(\frac{x}{2^{-2-j} \delta}\right)$. When $2^{-j} \delta \leq|x|<2^{1-j} \delta$, we obtain

$$
\begin{aligned}
\left|\partial_{i}\left(u_{n}-\Phi_{n}\right)(x)\right| & =\left|\int \frac{x_{i}-y_{i}}{|x-y|^{2}}\left(g_{n}(y)-\psi \tau_{n}(y)\right) d y\right| \\
& \leq \int \frac{\left|\psi A\left(u_{n}\right)\left(d u_{n}, d u_{n}\right)+2 \nabla \psi \nabla u_{n}+u_{n} \Delta \psi\right|(y)}{|x-y|} d y \\
& \leq \int \frac{\left|\psi A\left(u_{n}\right)(y)\right|}{|x-y|} d y+C \int_{1<|y|<2}\left(\left|\nabla u_{n}\right|+\left|u_{n}\right|\right)(y) d y \\
& \leq \int \frac{\left|\varphi_{j} A\left(u_{n}\right)(y)\right|}{|x-y|} d y+\int \frac{\left|\left(\psi-\varphi_{j}\right) A\left(u_{n}\right)(y)\right|}{|x-y|} d y+C \\
& \leq \int \frac{\left|\varphi_{j} A\left(u_{n}\right)(y)\right|}{|x-y|} d y+\frac{\int\left|A\left(u_{n}\right)(y)\right| d y}{|x|}+C \\
& \leq \int \frac{\left|\varphi_{j} A\left(u_{n}\right)(y)\right|}{|x-y|} d y+\frac{C}{|x|} .
\end{aligned}
$$


When $\delta>0$ is small enough and $n$ is big enough, for any $j>0$, we claim that

$$
\left\|\varphi_{j} A\left(u_{n}\right)\right\|_{p /(2-p)} \leq C\left(2^{-j} \delta\right)^{-4(p-1) / p},
$$

where the constant $C$ depends only on $p, \Lambda$, the bubble $w$ and the target manifold $N$.

Take $\delta>0$ and $R(w)$ that depends on $w$ such that

$$
E\left(u, D_{8 \delta}\right) \leq \frac{1}{8} \epsilon_{N}^{2} \quad \text { and } \quad E\left(w, \mathbb{R}^{2} \backslash D_{R(w)}\right) \leq \frac{1}{8} \epsilon_{N}^{2} .
$$

The standard blow-up analysis (see [Ding and Tian 1995]) shows that for any $j$ with $8 r_{n} R(w) \leq 2^{-j} \delta$ and $n$ big enough, we have

$$
E\left(u_{n}, D_{2^{4-j} \delta} \backslash D_{2^{-3-j} \delta}\right) \leq \frac{1}{3} \epsilon_{N}^{2} .
$$

By (3-1), when $2^{-j} \delta<r_{n} / 16$, we get

$$
E\left(u_{n}, D_{2^{4-j} \delta} \backslash D_{2^{-3-j} \delta}\right) \leq \frac{1}{4} \epsilon_{N}^{2} .
$$

So when $2^{-j} \delta<r_{n} / 16$ or $2^{-j} \delta \geq 8 r_{n} R(w)$, by Lemma 2 , we see that

$$
\begin{aligned}
& \left\|\varphi_{j} A\left(u_{n}\right)\right\|_{p /(2-p)} \leq C\left\|\nabla u_{n}\right\|_{L^{2 p /(2-p)}\left(D_{2^{3-j} \delta} \backslash D_{2^{-2-j} \delta}\right)}^{2} \\
& \leq C\left\|u_{n}-\bar{u}_{n, j}\right\|_{W^{2, p}\left(D_{2^{3-j} \delta} \backslash D_{2^{-2-j} \delta}\right)}^{2} \\
& \leq C\left[\left(2^{-j} \delta\right)^{-4 \frac{p-1}{p}}\left\|\nabla u_{n}\right\|_{L^{2}\left(D_{2^{4-j} \delta} \backslash D_{2^{-4-j} \delta}\right)}^{2}+\left\|\tau\left(u_{n}\right)\right\|_{p}^{2}\right] \\
& \leq C\left(2^{-j} \delta\right)^{-4 \frac{p-1}{p}} \text {, }
\end{aligned}
$$

where $\bar{u}_{n, j}$ is the mean of $u_{n}$ on $D_{2^{3-j} \delta} \backslash D_{2^{-2-j} \delta}$.

On the other hand, when $r_{n} / 16 \leq 2^{-j} \delta \leq 8 r_{n} R(w)$, we can find no more than $C R(w)^{2}$ balls with radius $r_{n} / 2$ to cover $D_{2^{3-j} \delta} \backslash D_{2^{-2-j} \delta}$, that is,

$$
D_{2^{3-j} \delta} \backslash D_{2^{-2-j} \delta} \subset \bigcup_{i=1}^{m} D\left(y_{i}, \frac{1}{2} r_{n}\right) .
$$

Set $B_{i}=D\left(y_{i}, \frac{1}{2} r_{n}\right)$ and $2 B_{i}=D\left(y_{i}, r_{n}\right)$. By (3-1), for any $i$ with $i \leq m$ we have

$$
E\left(u_{n}, 2 B_{i}\right) \leq \frac{1}{4} \epsilon_{N}^{2} .
$$

Using Lemma 2 we obtain

$$
\begin{aligned}
\left\|\varphi_{j} A\left(u_{n}\right)\right\|_{p /(2-p)} & \leq C\left\|\nabla u_{n}\right\|_{L^{2 p /(2-p)}\left(D_{2^{3}-j} \backslash D_{2^{-2-j} \delta}\right)}^{2} \\
& \leq C\left(\sum_{i=1}^{m}\left\|\nabla u_{n}\right\|_{L^{2 p /(2-p)}\left(B_{i}\right)}^{2 p /(2-p)}\right)^{(2-p) / p} \\
& \leq C \sum_{i=1}^{m}\left\|\nabla u_{n}\right\|_{L^{2 p /(2-p)}\left(B_{i}\right)}^{2}
\end{aligned}
$$




$$
\begin{aligned}
& \leq C \sum_{i=1}^{m}\left\|u_{n}-\bar{u}_{n, i}\right\|_{W^{2, p}\left(B_{i}\right)}^{2} \\
& \leq C \sum_{i=1}^{m}\left(\left(r_{n}\right)^{-4(p-1) / p}\left\|\nabla u_{n}\right\|_{L^{2}\left(2 B_{i}\right)}^{2}+\left\|\tau\left(u_{n}\right)\right\|_{p}^{2}\right) \\
& \leq C m\left(\left(2^{-j} \delta\right)^{-4(p-1) / p}+1\right) \\
& \leq C\left(2^{-j} \delta\right)^{-4(p-1) / p},
\end{aligned}
$$

where $\bar{u}_{n, i}$ is the mean of $u_{n}$ over $B_{i}$ and the constant $C$ depends only on $p, \Lambda$, the bubble $w$ and the target manifold $N$. So we have proved (3-11).

By (3-10) and (3-11), when $p>1$ we get

$$
\begin{aligned}
& \int_{D_{\delta}}\left|\tau_{n}\right|\left|\nabla\left(u_{n}-\Phi_{n}\right)(x)\right||x| \ln \frac{1}{|x|} d x \\
& \leq \sum_{j=1}^{\infty} \int_{2^{-j} \delta<|x|<2^{1-j} \delta}\left|\tau_{n}\right|\left|\nabla\left(u_{n}-\Phi_{n}\right)(x)\right||x| \ln \frac{1}{|x|} d x \\
& \leq C \sum_{j=1}^{\infty} \int_{2^{-j} \delta<|x|<2^{1-j} \delta}\left|\tau_{n}\right|\left(\frac{1}{|x|}+\int \frac{\left|\varphi_{j} A\left(u_{n}\right)(y)\right|}{|x-y|} d y\right)|x| \ln \frac{1}{|x|} d x \\
& \leq C\left(\int_{D_{\delta}}\left|\tau_{n}\right| \ln \frac{1}{|x|} d x\right. \\
& \left.+\sum_{j=1}^{\infty} \int_{2^{-j} \delta<|x|<2^{1-j} \delta}\left|\tau_{n}\right|\left(\int \frac{\left|\varphi_{j} A\left(u_{n}\right)(y)\right|}{|x-y|} d y\right)|x| \ln \frac{1}{|x|} d x\right) \\
& \leq C\left(\left\|\ln \frac{1}{|\cdot|}\right\|_{L^{p /(p-1)}\left(D_{\delta}\right)}+\sum_{j=1}^{\infty} 2^{-j} \delta \ln \frac{2^{j}}{\delta}\left\|\int \frac{\left|\varphi_{j} A\left(u_{n}\right)(y)\right|}{|\cdot-y|} d y\right\|_{\frac{p}{p-1}}\right) \\
& \times\left\|\tau_{n}\right\|_{p} \\
& \leq C\left(\delta^{2}\left(\ln \frac{1}{\delta}\right)^{1 /(p-1)}+\sum_{j=1}^{\infty} 2^{-j} \delta \ln \frac{2^{j}}{\delta}\left\|\varphi_{j} A\left(u_{n}\right)\right\|_{2 p /(3 p-2)}\right) .
\end{aligned}
$$

Here we use the fact that the fraction integral operator $I(f)=\frac{1}{|\cdot|} * f$ is bounded from $L^{q}\left(\mathbb{R}^{2}\right)$ to $L^{2 q /(2-q)}\left(\mathbb{R}^{2}\right)$ for $1<q<2$.

When $p \geq \frac{6}{5}$, that is, when $2 p /(3 p-2) \leq p /(2-p)$, by (3-11) we have

$$
\begin{aligned}
\left\|\varphi_{j} A\left(u_{n}\right)\right\|_{\frac{2 p}{3 p-2}} & \leq C\left(2^{-j} \delta\right)^{\frac{5 p-6}{p}}\left\|\varphi_{j} A\left(u_{n}\right)\right\|_{\frac{p}{2-p}} \\
& \leq C\left(2^{-j} \delta\right)^{\frac{5 p-6}{p}-\frac{4(p-1)}{p}} \leq C\left(2^{-j} \delta\right)^{-\frac{2-p}{p}} .
\end{aligned}
$$


From (3-12) and (3-13) we get

$$
\begin{aligned}
\int_{D_{\delta}}\left|\tau_{n}\right| \mid & \nabla \\
& \leq C\left(u_{n}-\Phi_{n}\right)(x)|| x \mid \ln \frac{1}{|x|} d x \\
& \leq C\left(\delta+\sum_{j=1}^{\infty} 2^{-j} \delta \ln \frac{2^{j}}{\delta}\left(2^{-j} \delta\right)^{-\frac{1}{p-1}}+\sum_{j=1}^{\infty} 2^{-j} \delta \ln \frac{2^{j}}{\delta}\left\|\varphi_{j} A\left(u_{n}\right)\right\|_{\frac{2 p}{3 p-2}}\right) \\
& \leq C\left(\delta+\delta^{\frac{2(p-1)}{p}} \ln \frac{1}{\delta}\right) \leq C \delta^{\frac{p-1}{p}} .
\end{aligned}
$$

It is clear that (3-9) and (3-14) imply that

$$
\left|\int_{D_{\delta}}\left(\left|\partial_{r} u_{n}\right|^{2}-r^{-2}\left|\partial_{\theta} u_{n}\right|^{2}\right) d x\right| \leq C \delta^{(p-1) / p} .
$$

This concludes the proof.

Now we use these lemmas to prove the energy identity. Note that $w$ is harmonic. From Lemma 4 we see that $\int_{D_{R}}\left(\left|\partial_{r} w\right|^{2}-r^{-2}\left|\partial_{\theta} w\right|^{2}\right) d x=0$ for any $R>0$. It is easy to see that

$$
\begin{aligned}
\lim _{R \rightarrow \infty} \lim _{n \rightarrow \infty}\left|\int_{D_{r_{n} R}}\left(\left|\partial_{r} u_{n}\right|^{2}-r^{-2}\left|\partial_{\theta} u_{n}\right|^{2}\right) d x\right| & =\lim _{R \rightarrow \infty}\left|\int_{D_{R}}\left(\left|\partial_{r} w\right|^{2}-r^{-2}\left|\partial_{\theta} w\right|^{2}\right) d x\right| \\
& =0 .
\end{aligned}
$$

Letting $\delta \rightarrow 0$ in (3-15), we obtain

$$
\text { (3-16) } \begin{aligned}
\lim _{\delta \rightarrow 0} \lim _{R \rightarrow \infty} \lim _{n \rightarrow \infty} \mid & \int_{D_{\delta} \backslash D_{r_{n} R}}\left(\left|\partial_{r} u_{n}\right|^{2}-r^{-2}\left|\partial_{\theta} u_{n}\right|^{2}\right) d x \mid \\
\leq & \lim _{\delta \rightarrow 0} \lim _{n \rightarrow \infty}\left|\int_{D_{\delta}}\left(\left|\partial_{r} u_{n}\right|^{2}-r^{-2}\left|\partial_{\theta} u_{n}\right|^{2}\right) d x\right| \\
\quad & \quad \lim _{R \rightarrow \infty} \lim _{n \rightarrow \infty}\left|\int_{D_{r_{n} R}}\left(\left|\partial_{r} u_{n}\right|^{2}-r^{-2}\left|\partial_{\theta} u_{n}\right|^{2}\right) d x\right| \\
& =0 .
\end{aligned}
$$

Using Lemma 3 we obtain that the normal energy also vanishes on the neck domain, so the energy identity is proved.

\section{Neckless property}

In this section we use the method in [Qing and Tian 1997] to prove the neckless property during blowing up. 
For any $\epsilon>0$, take $\delta, R$ such that

$$
E\left(u, D_{4 \delta}\right)+E\left(w, \mathbb{R}^{2} \backslash D_{R}\right)+\delta^{4(p-1) / p}<\epsilon^{2} .
$$

Suppose $r_{n} R=2^{-j_{n}}, \delta=2^{-j_{0}}$. When $n$ is big enough, the standard blow-up analysis shows that for any $j_{0} \leq j \leq j_{n}$,

$$
E\left(u_{n}, D_{2^{1-j}} \backslash D_{2^{-j}}\right)<\epsilon^{2} .
$$

For any $j_{0}<j<j_{n}$, set $L_{j}=\min \left\{j-j_{0}, j_{n}-j\right\}$. Now we estimate the norm $\left\|\nabla u_{n}\right\|_{L^{2}\left(P_{j}\right)}$. Set $P_{j, t}=D_{2^{t-j}} \backslash D_{2^{-t-j}}$ and take $h_{n, j, t}$ similar to $h_{n}$ in the last section, but

$$
h_{n, j, t}\left(2^{ \pm t-j}\right)=\frac{1}{2 \pi} \int_{S^{1}} u_{n}\left(2^{ \pm t-j}, \theta\right) d \theta .
$$

By an argument similar to the one used in deriving (3-4), we have, for $0<t \leq L_{j}$,

$$
\begin{aligned}
& \int_{P_{j, t}} r^{-2}\left|\partial_{\theta} u_{n}\right|^{2} d x \\
& \quad \leq C \epsilon\left(\int_{P_{j, t}}\left|\nabla u_{n}\right|^{2} d x+\left(2^{t-j}\right)^{\frac{2(p-1)}{p}}\right)+\int_{\partial P_{j, t}}\left|u_{n}-h_{n, j, t}\right|\left|\nabla u_{n}\right| d s .
\end{aligned}
$$

Set $f_{j}(t)=\int_{P_{j, t}}\left|\nabla u_{n}\right|^{2} d x$, a simple computation shows that

$$
f_{j}^{\prime}(t)=\ln 2\left(2^{t-j} \int_{\left\{2^{t-j}\right\} \times S^{1}}\left|\nabla u_{n}\right|^{2} d s+2^{-t-j} \int_{\left\{2^{-t-j}\right\} \times S^{1}}\left|\nabla u_{n}\right|^{2} d s\right) .
$$

Combining that $h_{n, j, t}$ is independent of $\theta$ and $h_{n, j, t}$ is the mean value of $u_{n}$ at the two components of $\partial P_{j, t}$ with the Poincaré inequality yields that

$$
\begin{aligned}
\int_{\partial P_{j, t}} \mid u_{n}- & h_{n, j, t}|| \nabla u_{n} \mid d s \\
& =\int_{\left\{2^{t-j}\right\} \times S^{1}}\left|u_{n}-h_{n, j, t}\right|\left|\nabla u_{n}\right| d s+\int_{\left\{2^{-t-j}\right\} \times S^{1}}\left|u_{n}-h_{n, j, t}\right|\left|\nabla u_{n}\right| d s \\
& \leq\left(\int_{\left\{2^{t-j}\right\} \times S^{1}}\left|u_{n}-h_{n, j, t}\right|^{2} d s\right)^{\frac{1}{2}}\left(\int_{\left\{2^{t-j}\right\} \times S^{1}}\left|\nabla u_{n}\right|^{2} d s\right)^{\frac{1}{2}} \\
& \left.\quad+\left(\int_{\left\{2^{-t-j}\right\} \times S^{1}}\left|u_{n}-h_{n, j, t}\right|^{2} d s\right)^{\frac{1}{2}}\left(\int_{\left\{2^{-t-j}\right\} \times S^{1}}\left|\nabla u_{n}\right|^{2} d s\right)^{\frac{1}{2}}\left|\nabla u_{n}\right|^{2} d s+2^{-t-j} \int_{\left\{2^{-t-j}\right\} \times S^{1}}\left|\nabla u_{n}\right|^{2} d s\right) \\
& \leq C\left(2^{t-j} \int_{\left\{2^{t-j}\right\} \times S^{1}}\right. \\
& \leq C f_{j}^{\prime}(t) .
\end{aligned}
$$

On the other hand, by a similar argument as we made to obtain (3-15), we get 


$$
\begin{aligned}
& \left|\int_{P_{j, t}}\left(\left|\partial_{r} u_{n}\right|^{2}-r^{-2}\left|\partial_{\theta} u_{n}\right|^{2}\right) d x\right| \\
& \quad \leq C\left(\left(2^{t-j}\right)^{\frac{p-1}{p}}+\left(2^{-t-j}\right)^{\frac{p-1}{p}}\right) \leq C\left(2^{t-j}\right)^{\frac{p-1}{p}} .
\end{aligned}
$$

Since $|\nabla u|^{2}=\left|\partial_{r} u\right|^{2}+r^{-2}\left|\partial_{\theta} u\right|^{2}=2 r^{-2}\left|\partial_{\theta} u\right|^{2}+\left(\left|\partial_{r} u\right|^{2}-r^{-2}\left|\partial_{\theta} u\right|^{2}\right)$, by (4-1) and (4-2) we have

$$
\begin{aligned}
f_{j}(t) & \leq 2 \int_{P_{j, t}} r^{-2}\left|\partial_{\theta} u_{n}\right| d x+\left|\int_{P_{j, t}}\left(\left|\partial_{r} u_{n}\right|^{2}-r^{-2}\left|\partial_{\theta} u_{n}\right|^{2}\right) d x\right| \\
& \leq C \epsilon\left(f_{j}(t)+\left(2^{t-j}\right)^{\frac{2(p-1)}{p}}\right)+C f_{j}^{\prime}(t)+C\left(2^{t-j}\right)^{\frac{p-1}{p}} \\
& \leq C\left(\epsilon f_{j}(t)+2^{-\frac{(p-1) j}{p}} 2^{\frac{(p-1) t}{p}}+f_{j}^{\prime}(t)\right) .
\end{aligned}
$$

Take $\epsilon$ small enough and set $\epsilon_{p}=\frac{p-1}{p} \ln 2$, then for some positive constant $C$ big enough we get

$$
f_{j}^{\prime}(t)-\frac{1}{C} f_{j}(t)+C e^{-\epsilon_{p} j} e^{\epsilon_{p} t} \geq 0 .
$$

We may assume that $\epsilon_{p}>1 / C$, then we have

$$
\left(e^{-t / C} f_{j}(t)\right)^{\prime}+C e^{-\epsilon_{p} j} e^{\left(\epsilon_{p}-1 / C\right) t} \geq 0 .
$$

Integrating this inequality over $\left[2, L_{j}\right]$ gives

$$
\begin{aligned}
f_{j}(2) & \leq C\left(e^{-L_{j} / C} f_{j}\left(L_{j}\right)+e^{-\epsilon_{p} j} \int_{1}^{L_{j}} e^{\left(\epsilon_{p}-1 / C\right) t} d t\right) \\
& \leq C\left(e^{-L_{j} / C} f_{j}\left(L_{j}\right)+e^{-\epsilon_{p} j} e^{\left(\epsilon_{p}-1 / C\right) L_{j}}\right) .
\end{aligned}
$$

Note that $j \geq L_{j}$, so

$$
f_{j}(2) \leq C\left(e^{-L_{j} / C} f_{j}\left(L_{j}\right)+e^{-j / C}\right) .
$$

Since the energy identity was proved in the last section, we can take $\delta$ small such that the energy on the neck domain is less than $\epsilon^{2}$, which implies that $f_{j}\left(L_{j}\right)<\epsilon^{2}$. So we get

$$
f_{j}(2) \leq C\left(e^{-L_{j} / C} \epsilon^{2}+e^{-j / C}\right) .
$$

Using Lemma 2 on the domain $P_{j}=D_{2^{1-j}} \backslash D_{2^{-j}}$ when $j<j_{n}$, we obtain

$$
\begin{aligned}
\left\|u_{n}\right\|_{O s c\left(P_{j}\right)} & \leq C\left(\left\|\nabla u_{n}\right\|_{L^{2}\left(P_{j-1} \cup P_{j} \cup P_{j+1}\right)}+2^{\frac{2(1-p) j}{p}}\left\|\tau\left(u_{n}\right)\right\|_{p}\right) \\
& \leq C\left(f_{j}(2)+e^{-2 \epsilon_{p} j}\right) .
\end{aligned}
$$


Summing over $j$ from $j_{0}$ to $j_{n}$ yields

$$
\begin{aligned}
\left\|u_{n}\right\|_{O s c}\left(D_{\delta} \backslash D_{2 r_{n} R}\right) & \leq \sum_{j=j_{0}}^{j_{n}}\left\|u_{n}\right\|_{O s c\left(P_{j}\right)} \\
& \leq C \sum_{j=j_{0}}^{j_{n}}\left(f_{j}(2)+e^{-2 \epsilon_{p} j}\right) \\
& \leq C \sum_{j=j_{0}}^{j_{n}}\left(e^{-L_{j} / C} \epsilon^{2}+e^{-j / C}+e^{-2 \epsilon_{p} j}\right) \\
& \leq C\left(\sum_{i=0}^{\infty} e^{-i / C} \epsilon^{2}+\sum_{j=j_{0}}^{\infty} e^{-j / C}\right) \\
& \leq C\left(\epsilon^{2}+e^{-j_{0} / C}\right) \leq C\left(\epsilon^{2}+\delta^{1 / C}\right) .
\end{aligned}
$$

Here we used the assumption that $\epsilon_{p}>1 / C$. So we have proved that there is no neck during the blowing up.

\section{References}

[Ding and Tian 1995] W. Ding and G. Tian, "Energy identity for a class of approximate harmonic maps from surfaces”, Comm. Anal. Geom. 3:3-4 (1995), 543-554. MR 97e:58055 Zbl 0855.58016

[Li and Zhu 2011] J. Li and X. Zhu, "Small energy compactness for approximate harmomic mappings”, Commun. Contemp. Math. 13:5 (2011), 741-763. MR 2847227 Zbl 1245.58008

[Lin and Wang 1998] F. Lin and C. Wang, "Energy identity of harmonic map flows from surfaces at finite singular time", Calc. Var. Partial Differential Equations 6:4 (1998), 369-380. MR 99k:58047 Zbl 0908.58008

[Lin and Wang 2002] F. Lin and C. Wang, "Harmonic and quasi-harmonic spheres, II", Comm. Anal. Geom. 10:2 (2002), 341-375. MR 2003d:58029 Zbl 1042.58005

[Parker 1996] T. H. Parker, "Bubble tree convergence for harmonic maps", J. Differential Geom. 44:3 (1996), 595-633. MR 98k:58069 Zbl 0874.58012

[Qing 1995] J. Qing, "On singularities of the heat flow for harmonic maps from surfaces into spheres”, Comm. Anal. Geom. 3:1-2 (1995), 297-315. MR 97c:58154 Zbl 0868.58021

[Qing and Tian 1997] J. Qing and G. Tian, "Bubbling of the heat flows for harmonic maps from surfaces”, Comm. Pure Appl. Math. 50:4 (1997), 295-310. MR 98k:58070 Zbl 0879.58017

[Topping 2004a] P. Topping, "Repulsion and quantization in almost-harmonic maps, and asymptotics of the harmonic map flow", Ann. of Math. (2) 159:2 (2004), 465-534. MR 2005g:58029 Zbl 1065.58007

[Topping 2004b] P. Topping, "Winding behaviour of finite-time singularities of the harmonic map heat flow”, Math. Z. 247:2 (2004), 279-302. MR 2004m:53120 Zbl 1067.53055

[Wang 1996] C. Wang, "Bubble phenomena of certain Palais-Smale sequences from surfaces to general targets”, Houston J. Math. 22:3 (1996), 559-590. MR 98h:58053 Zbl 0879.58019

[Zhu 2012] X. Zhu, "No neck for approximate harmonic maps to the sphere", Nonlinear Anal. 75:11 (2012), 4339-4345. MR 2921993 Zbl 1243.58011 
Received October 1, 2011. Revised May 3, 2012.

LI JIAYU

WU WENJUN KEY LABORATORY

SCHOOL OF MathematiCAL SCIENCES

UNIVERSITY OF SCIENCE AND TECHNOLOGY OF CHINA

96 JINZHAI ROAD

HEFEI, 230026

CHINA

and

AMSS, Chinese ACADEMy OF SCIENCES

BEIJING, 100190

CHINA

jiayuli@ustc.edu.cn

ZHU XIANGRONG

DEPARTMENT OF MATHEMATICS

ZHEJIANG NORMAL UNIVERSITY

688 YINGBIN ROAD

JINHUA, 321004

CHINA

zxr@zjnu.cn 


\title{
PACIFIC JOURNAL OF MATHEMATICS
}

\author{
http://pacificmath.org \\ Founded in 1951 by \\ E. F. Beckenbach (1906-1982) and F. Wolf (1904-1989)
}

\section{EDITORS}

V. S. Varadarajan (Managing Editor)

Department of Mathematics

University of California

Los Angeles, CA 90095-1555

pacific@math.ucla.edu

Vyjayanthi Chari

Department of Mathematics

University of California

Riverside, CA 92521-0135

chari@math.ucr.edu

\section{Robert Finn}

Department of Mathematics Stanford University

Stanford, CA 94305-2125

finn@math.stanford.edu

Kefeng Liu

Department of Mathematics

University of California

Los Angeles, CA 90095-1555

liu@math.ucla.edu
Darren Long

Department of Mathematics

University of California

Santa Barbara, CA 93106-3080

long@math.ucsb.edu

Jiang-Hua Lu

Department of Mathematics

The University of Hong Kong

Pokfulam Rd., Hong Kong jhlu@maths.hku.hk

Alexander Merkurjev

Department of Mathematics

University of California

Los Angeles, CA 90095-1555

merkurev@math.ucla.edu
Sorin Popa

Department of Mathematics University of California

Los Angeles, CA 90095-1555 popa@math.ucla.edu

Jie Qing

Department of Mathematics

University of California

Santa Cruz, CA 95064

qing@cats.ucsc.edu

Jonathan Rogawski

Department of Mathematics

University of California

Los Angeles, CA 90095-1555

jonr@math.ucla.edu

\section{PRODUCTION}

pacific@math.berkeley.edu

\section{SUPPORTING INSTITUTIONS}

ACADEMIA SINICA, TAIPEI

CALIFORNIA INST. OF TECHNOLOGY INST. DE MATEMÁTICA PURA E APLICADA KEIO UNIVERSITY

MATH. SCIENCES RESEARCH INSTITUTE NEW MEXICO STATE UNIV.

OREGON STATE UNIV.

\author{
STANFORD UNIVERSITY \\ UNIV. OF BRITISH COLUMBIA \\ UNIV. OF CALIFORNIA, BERKELEY \\ UNIV. OF CALIFORNIA, DAVIS \\ UNIV. OF CALIFORNIA, LOS ANGELES \\ UNIV. OF CALIFORNIA, RIVERSIDE \\ UNIV. OF CALIFORNIA, SAN DIEGO \\ UNIV. OF CALIF., SANTA BARBARA
}

\author{
UNIV. OF CALIF., SANTA CRUZ \\ UNIV. OF MONTANA \\ UNIV. OF OREGON \\ UNIV. OF SOUTHERN CALIFORNIA \\ UNIV. OF UTAH \\ UNIV. OF WASHINGTON \\ WASHINGTON STATE UNIVERSITY
}

These supporting institutions contribute to the cost of publication of this Journal, but they are not owners or publishers and have no responsibility for its contents or policies.

See inside back cover or pacificmath.org for submission instructions.

The subscription price for 2012 is US \$420/year for the electronic version, and \$485/year for print and electronic.

Subscriptions, requests for back issues from the last three years and changes of subscribers address should be sent to Pacific Journal of Mathematics, P.O. Box 4163, Berkeley, CA 94704-0163, U.S.A. Prior back issues are obtainable from Periodicals Service Company, 11 Main Street, Germantown, NY 12526-5635. The Pacific Journal of Mathematics is indexed by Mathematical Reviews, Zentralblatt MATH, PASCAL CNRS Index, Referativnyi Zhurnal, Current Mathematical Publications and the Science Citation Index.

The Pacific Journal of Mathematics (ISSN 0030-8730) at the University of California, c/o Department of Mathematics, 969 Evans Hall, Berkeley, CA 94720-3840, is published monthly except July and August. Periodical rate postage paid at Berkeley, CA 94704, and additional mailing offices. POSTMASTER: send address changes to Pacific Journal of Mathematics, P.O. Box 4163, Berkeley, CA 94704-0163.

PJM peer review and production are managed by EditFLOW ${ }^{\mathrm{TM}}$ from Mathematical Sciences Publishers.

PUBLISHED BY PACIFIC JOURNAL OF MATHEMATICS

at the University of California, Berkeley 94720-3840

A NON-PROFIT CORPORATION

Typeset in LATEX

Copyright $(02012$ by Pacific Journal of Mathematics 


\section{PACIFIC JOURNAL OF MATHEMATICS}

Volume $260 \quad$ No. $1 \quad$ November 2012

The decomposition of global conformal invariants: Some technical proofs II

SPYROS ALEXAKIS

On deformation quantizations of hypertoric varieties

GWYN BELLAMY and TOSHIRO KUWABARA

Almost factoriality of integral domains and Krull-like domains

GYU Whan CHANG, HWANKOO KIM and JUNG WOOK LIM

Singularities of free group character varieties

CARLOS FLORENTINO and SEAN LAWTON

Energy identity for the maps from a surface with tension field bounded 181 in $L^{p}$

LI JIAYU and ZHU XIANGRONG

Remarks on some isoperimetric properties of the $k-1$ flow

YU-CHU LIN and DONG-Ho TSAI

Demystifying a divisibility property of the Kostant partition function 215

KAROLA MÉSZÁros

Exceptional Lie algebras, SU(3), and Jordan pairs

PIERO TRUINI

Lower estimate of Milnor number and characterization of isolated homogeneous hypersurface singularities

Stephen S.-T. Yau and HuAiqing Zuo 